\title{
Current Approach to Acute and Chronic Airway Disease
}

\author{
Philippe A. Grenier and Jeffrey P. Kanne
}

\section{Learning Objectives}

- To remind the basic principles of volumetric thincollimation CT technique permitting to obtain an optimized evaluation of central and peripheral airway

- To know the CT features of most of the diseases involving the trachea and central bronchi

- To know the diagnostic criteria to identify the presence, distribution, and extent of bronchiectasis

- To know the direct and indirect CT features suggestive of inflammation, infection, and obstruction of small airways

\subsection{CT Acquisition and Post-processing Techniques [1, 2]}

MDCT acquisition is performed through the entirety of the lungs at full-suspended inspiration, using thin collimation $(0.6-1.5 \mathrm{~mm})$ without administration of contrast material. Axial images are reconstructed with thin-slice thickness $(0.8-1.5 \mathrm{~mm})$ and overlap (50\% overlap is optimal). Complementary MDCT acquisition at full continuous forced expiration using reduced technique $(120 \mathrm{Kv}-20-40 \mathrm{mAs})$ is often recommended and particularly useful for assessing tracheobronchial collapsibility and expiratory air trapping.

Interpretation is best performed on a PACS workstation. Evaluation of the overlapped thin-section axial images in cine-mode allows the bronchial divisions to be easily followed from the carina to the smallest peripheral bronchi visible on

P. A. Grenier $(\bowtie)$

Department Radiology, Sorbonne Université - Hôpital Pitié-

Salpêtrière, Paris, France

\section{J. P. Kanne}

Department of Radiology, University of Wisconsin School

of Medicine and Public Health, Madison, WI, USA

e-mail:kanne@wisc.edu
CT. Real-time manipulation of the data set allows the radiologist to select the optimal plane to better depict the distribution and extent of airway abnormalities. Furthermore, multiplanar reformations with use of minimum and maximum intensity projections can be helpful to display airway abnormalities. Minimum intensity projection (minIP) can highlight airway dilation, diverticula, fistulas, and the extent of expiratory air trapping and emphysema. Maximum intensity projection (MIP) accentuates foci of mucoid impaction and small centrilobular nodules and tree-in-bud opacities. Volume rendering techniques (CT bronchography) can segment the airway lumen-wall interface and can be useful to detect subtle changes in airway calibre and to facilitate understanding of complex tracheobronchial abnormalities. Virtual bronchoscopy provides an internal rendering of the tracheobronchial inner surface and can better depict subtle mucosal abnormalities such as neoplasia and granulomatous disease.

\subsection{Tracheobronchial Tumours (Tables 6.1, 6.2, 6.3, and 6.4) [3-6]}

\subsubsection{Primary Malignant Neoplasms}

Primary malignant neoplasms are rare in the trachea compared to the laryngeal or bronchial cancers. The most frequent histological types are squamous cell carcinoma and adenoid cystic carcinoma, accounting for nearly $80 \%$ of all tracheal neoplasms. Adenoid cystic carcinoma (sialadenoid neoplasm) is a low-grade malignancy that is not associated with cigarette smoking. It occurs in patients in their 40s without any sex predilection. In the central airways, adenoid cystic carcinoma has a propensity to infiltrate the wall of the airways with submucosal extension manifesting as a sessile, polypoid, annular, or diffuse infiltration. The inner surface is smooth and regular. An extraluminal growth, visible on CT scan, is a common feature. Squamous cell carcinoma is the most common primary malignancy of the trachea primarily occurring in older men with a history of cigarette smoking. 
Table 6.1 Luminal filling defect

- Benign tracheobronchial neoplasm

- Malignant primary tracheobronchial neoplasm

- Metastasis

- Mucus secretions

- Foreign body

- Broncholithiasis

Table 6.2 Multinodular appearance of the inner surface of the airway

- Granulomatosis with polyangiitis

- Tracheobronchial metastases (haematogenous spread)

- Respiratory papillomatosis

- Adenoid cystic carcinoma (multicentric)

- Tracheobronchial amyloidosis

- Tracheobronchopathia osteochondroplastica

Table 6.3 Focal tracheobronchial narrowing

- Posttraumatic strictures

- Postinfectious stenoses (e.g. tuberculosis)

- Tracheobronchial neoplasms (primary and secondary malignant)

- Granulomatosis with polyangiitis (can be multifocal)

- Sarcoidosis

- Inflammatory bowel disease

- Extrinsic compression

Table 6.4 Diffuse tracheobronchial wall thickening

- Infectious tracheobronchitis (TB, aspergillosis)

- Relapsing polychondritis ${ }^{\mathrm{a}}$

- Tracheobronchial amyloidosis

- Tracheobronchopathia osteochrondroplastica ${ }^{\mathrm{a}}$

- Tracheobronchitis associated with inflammatory bowel disease

- Sarcoidosis (rare manifestation)

${ }^{\mathrm{a}}$ Calcification frequently present and posterior membrane characteristically spared

On CT, the tumour appears as a polypoid intraluminal mass or as an eccentric irregular wall thickening with an irregular surface. The tumour has a tendency to spread to adjacent mediastinal lymph nodes and to directly invade the mediastinum. Carcinoid tumour is a low-grade malignant neuroendocrine neoplasm representing 1-2\% of primary lung neoplams. It appears on CT scan as a well-circumscribed polypoid mass that protrudes into the airway lumen. Segmental or lobar atelectasis and obstructive pneumonitis, as well as foci of calcification are present in 30\% of cases. Marked homogeneous early contrast enhancement of an endobronchial nodule reflecting the high vascularity of this neoplasm may be present. Mucoepidermoid carcinoma is a rare neoplasm that originates from the minor salivary glands lining the tracheobronchial tree, occurring in young patients $(<40$ years old), found mainly in the segmental bronchi and resulting in airway obstruction. The typical CT appearance is a smooth, ovoid, or lobulated endobronchial mass with occasional punctuate calcifications and variable contrast enhancement.
Lymphoma of the trachea is rare, usually related to mucosaassociated lymphoid tissue. The CT appearance is indistinguishable from other neoplasms.

\subsubsection{Secondary Tracheobronchial Malignancy}

Direct invasion of the central airways by neoplasm of the thyroid, oesophagus, lung, and larynx is much more common that haematogenous metastases. CT shows the primary neoplasm and its extension by contiguity within the main airways (endoluminal mass, destruction of cartilage, and tracheobronchial-oesophageal fistula).

Many cancers have the potential to metastasize to the trachea and bronchi. Endotracheal or endobronchial metastases appear as endotracheal nodules or eccentric thickening of the airway wall or soft tissue attenuation with contrast enhancement.

\subsubsection{Benign Tracheobronchial Neoplasms}

Benign tracheal neoplasms are rare. On CT, they present as endoluminal masses confined within the tracheobronchial lumen without evidence of involvement of surrounding structures. Benign neoplasms are typically sharply marginated, round, and smaller than $2 \mathrm{~cm}$ in diameter. Histology mainly includes hamartomas, lipomas, leiomyomas, fibromas, chondromas, and schwannomas. Endobronchial hamartomas represent $30 \%$ of intrathoracic hamartomas. The presence of fat with or without calcification is diagnostic.

Respiratory papillomatosis (laryngotracheobronchial papillomatosis) is a neoplastic disease caused by human papillomavirus (HPV) infection transmitted from mother to child at birth or acquired from orogenital contact. Papillomas arise in the larynx and can involve trachea and proximal bronchi in up to $50 \%$ of cases. Involvement of the lungs is rare, occurring in fewer than $1 \%$ of patients, and manifests on imaging studies as multiple lung nodules and cavitary and cystic lesions in the parenchyma. Malignant transformation into squamous cell carcinoma is a rare but serious complication of respiratory papillomatosis.

\subsection{Nonneoplastic Tracheobronchial Disorders (Tables 6.2, 6.3, and 6.4) $[4,5,7]$}

\subsubsection{Posttraumatic Stenosis}

Posttraumatic strictures of the trachea are usually the result of ischemic injury from a cuffed endotracheal or tracheostomy tube or extrinsic neck trauma. The two principal sites of stenosis following intubation or tracheostomy tube are at 
the stroma or at the level of the endotracheal or tracheostomy tube balloon.

Postintubation stenosis extends for several centimetres

Key Point

- CT with multiplanar reformations clearly depicts the severity and length of the stricture.

and typically involves trachea above the level of the thoracic inlet. Posttracheostomy stenosis typically begins $1.0-1.5 \mathrm{~cm}$ distal to the inferior margin of the tracheostomy stoma and extends over $1.5-2.5 \mathrm{~cm}$. These strictures typically have an hourglass configuration with thickened tracheal wall. Less commonly the tracheal or bronchial stenosis may present as a thin membrane or granulation tissue protruding into the airway lumen.

\subsubsection{Infections}

A number of infections, both acute and more often chronic, may affect the trachea and proximal bronchi, resulting in both focal and diffuse airway disease. Subsequent fibrosis may result in localized airway narrowing. The most common causes of infectious tracheobronchitis are acute bacterial tracheitis in immunocompromised patients, tuberculosis, rhinoscleroma (Klebsiella rhinoscleromatis), and necrotizing invasive aspergillosis. On CT, the extent of irregular and circumferential tracheobronchial narrowing is readily apparent, and in some patients, an accompanying mediastinitis is evident, manifesting as infiltration of mediastinal fat. In the fibrotic or healed phase, the airway is narrowed but has a smooth and normal thickness wall. Occasionally, because of the presence of chronic fibrous or granulomatous cheilitis/ mediastinitis, tuberculosis of the trachea and/or proximal bronchi may mimic airway malignancy on CT.

\subsubsection{Granulomatosis with Polyangiitis (Wegener Granulomatosis)}

Inflammatory lesions may be present with or without subglottic or bronchial stenosis, ulcerations, and pseudotumours. Radiologic manifestations include thickening of the subglottic and proximal trachea with a smooth symmetric or asymmetric narrowing over variable length. Tracheal rings can become thickened and calcified. Cartilaginous erosions and ulcerations also may be seen. Stenosis can develop in any main, lobar, or segmental bronchus. Developing nodular or polypoid lesions can protrude into the airway lumen.

\subsubsection{Relapsing Polychondritis}

Relapsing polychondritis is a rare systemic autoimmune disease that affects cartilage at various sites, including the ears, nose, joints, and tracheobronchial tree. Symmetric subglottic stenosis is the most frequent manifestation in the chest. As the disease progresses, the distal trachea and bronchi may be involved. CT scans show smooth thickening of the airway wall associated with diffuse narrowing. In the early stages of the disease, the posterior wall of the trachea is spared, but in advanced disease, circumferential wall thickening can occur. Tracheobronchomalacia can develop as a result of weakening of cartilage, resulting in considerable luminal collapse on expiration. Marked destruction of the cartilaginous rings with fibrosis may cause stenosis.

\subsubsection{Amyloidosis}

Deposition of amyloid in the trachea and bronchi may occur in association with systemic amyloidosis or as an isolated process. CT scans show focal or, more commonly, diffuse thickening of the airway wall and narrowing of the lumen. Calcification may be present. Narrowing of the proximal bronchi can lead to distal atelectasis, bronchiectasis, or both with or without obstructive pneumonia.

\subsubsection{Tracheobronchopathia Osteochondroplastica (TO)}

TO is a rare disorder characterized by the presence of multiple cartilaginous nodules and bony submucosal nodules on the luminal surface of the trachea and proximal airways. TO involves males more frequently than females, and most patients are older than 50 years. Histologically, the nodules contain heterotopic bone, cartilage, and calcified acellular protein matrix. Because it contains no cartilage, the posterior wall of the trachea is spared. On CT, tracheal cartilages are thickened and show irregular calcifications. The nodules may protrude from the anterior and lateral walls into the lumen; they usually show foci of calcification.

\subsubsection{Sabre-Sheath Trachea}

Characterized by narrowing of the transverse diameter and increase of the sagittal diameter of the intrathoracic trachea, this deformity is almost always associated with COPD. The pathogenesis is unclear but likely results from altered mechanics from intrathoracic pressure changes associated with COPD. On radiographs and CT, the internal transverse diameter of the trachea is decreased to half or 
less than the corresponding sagittal diameter. The trachea usually shows a smooth inner margin but occasionally has a nodular contour. Calcification of the tracheal cartilage is common.

\subsubsection{Tracheobronchomegaly (Mounier-Kuhn Syndrome)}

This abnormality is characterized by abnormal dilation of the trachea and main bronchi and recurrent lower respiratory tract infection. The aetiology is not fully understood, but many patients have congenitally atrophic smooth muscle and elastin fibres. Mounier-Kuhn syndrome is often associated with tracheal diverticulosis and bronchiectasis. The subglottic trachea typically has a normal diameter, but the tracheal diameter expands toward the carina, often involving the central bronchi, as well. Atrophic mucosa prolapses between cartilage rings and gives the trachea a characteristically corrugated appearance. Corrugations may become exaggerated to form sacculations or diverticula. On CT a tracheal diameter of greater than $3 \mathrm{~cm}$ (measured $2 \mathrm{~cm}$ above the aortic arch) and diameter of $2.4 \mathrm{~cm}$ and $2.3 \mathrm{~cm}$ for the right and left bronchi, respectively, are diagnosing criteria. Additional findings include tracheal scalloping or diverticula (especially along the posterior membranous tracheal wall).

\subsubsection{Tracheobronchomalacia and Excessive Dynamic Airway Collapse $[8,9,10]$}

Tracheobronchomalacia (TBM) and excessive dynamic airway collapse (EDAC) may be responsible for expiratory central airway collapse (ECAC) which is an accepted term to describe the narrowing of central airway during expiration. TBM is characterized as a weakened or destroyed cartilage in the central airways resulting in expiratory airflow limitation. EDAC is characterized by excessive bulging of the posterior membrane inside the central airway lumen during expiration without cartilage collapse.

At CT, the diagnosis of EDAC is based on the narrowing of the lumen diameter by more than $70 \%$ on expiration compared with that on inspiration. The $50 \%$ reduction in airway cross-sectional area during forced expiration is inadequate to define abnormal collapse. No threshold of end-expiration collapse reliably predicts EDAC. Therefore dynamic expiratory imaging should be considered when there is high clinical suspicion for ECAC. In addition, dynamic expiratory multislice CT may offer feasible alternative to bronchoscopy in patients with suspected TBM or EDAC by showing complete relapse or collapse greater than $80 \%$ of airway lumen.

\subsubsection{Broncholithiasis}

Broncholithiasis is a rare condition characterized by erosion into or distortion of a bronchus from an adjacent calcified lymph node. The underlying abnormality is usually granulomatous lymphadenitis caused by Mycobacterium tuberculosis or fungi such as Histoplasma capsulatum. A few cases associated with silicosis have been reported. Calcified material in the bronchial lumen or bronchial distortion results in airway obstruction. This leads to collapse, obstructive pneumonitis, mucoid impaction, or bronchiectasis. Occasionally, patients will expectorate calcific fragments (lithoptysis). Broncholithiasis is recognized on CT by the presence of a calcified endobronchial or peribronchial lymph node, associated with bronchopulmonary complication related to obstruction in the absence of an associated soft tissue mass.

\subsection{Tracheobronchial Fistula, Dehiscence, and Diverticula (Table 6.5) $[2,11]$}

Peripheral bronchopleural fistulas are most commonly caused by necrotizing pneumonia or secondary to trauma. Nodobronchial and nodobronchoesophageal fistulas are characterized by the presence of gas in cavitated hilar or mediastinal lymph nodes adjacent to the airways and are usually caused by tuberculosis. Occasionally, congenital tracheal diverticula and tracheobronchoesophageal fistulas may not present until adulthood, especially the H-type.

Malignant neoplasia, particularly oesophageal, is the most common cause of tracheoesophageal fistula in adults. Infection and trauma are the most frequent nonmalignant causes.

MDCT has a high degree of sensitivity and specificity for depicting bronchial dehiscence occurring after lung transplantation. Bronchial dehiscence is seen as a bronchial wall defect at the anastomosis associated with extraluminal gas collections.

The cardiac bronchus is an uncommon airway anomaly characterized by a supernumerary bronchus arising from the inferomedial aspect of the mid bronchus intermedius. The cardiac bronchus typically is a blind-ending pouch but

Table 6.5 Tracheobronchial dehiscence, fistulas, and diverticula

- Tracheal or bronchial rupture

- Bronchial dehiscence occurring after lung transplantation

- Tracheal diverticula (tracheocele)

- Paratracheal cyst

- Accessory cardiac bronchus

- Multiple tracheobronchial diverticula

- Nodobronchial and nodobronchoesophageal fistulas

- Tracheoesophageal fistulas

- Bronchopleural fistulas 
occasionally supplies a very small number of pulmonary lobules. Most cardiac bronchi are clinically silent and thus are incidentally detected on chest CT. However, they may serve as a reservoir for retained secretions leading to chronic inflammation and hypervascularity, leading to recurrent episodes of infection or haemoptysis.

In smokers, bronchial diverticula or outpouchings may be seen as small airway collections in the wall of the main and lobar bronchi particularly well displayed on coronal reformations with minimal intensity projection. They express the fusion of multidepressions and dilations of the bronchial gland ducts forming a diverticulum, which herniates between and through the smooth muscle cellular bundles.

Paratracheal cysts are characterized by one or several cystic cavities present along the cervicothoracic part of the trachea. These cysts communicate with the tracheal lumen through a small wall defect visible on thin CT slices and located in the posterolateral angle of the trachea. The great majority is located on the right side.

\subsection{Bronchiectasis $[12,13]$}

This chronic condition characterized by local, irreversible dilation of bronchi, usually associated with inflammation, remains an important cause of haemoptysis and chronic sputum production.

Pathologically, bronchiectasis is classified into three subtypes, reflecting increasing severity of disease: cylindrical, characterized by relatively uniform airway dilatation; varicose, characterized by nonuniform and somewhat serpentine dilation; and cystic. As bronchiectasis progresses, the lung parenchyma distal to the affected airway increasingly collapses.

The CT findings of bronchial dilation include lack of tapering of bronchial lumina (the cardinal sign of bronchiectasis), internal diameter of bronchi greater than that of the adjacent pulmonary artery (signet ring sign), bronchi visible within $1 \mathrm{~cm}$ of the costal pleura or abutting the mediastinal pleura, and mucus-filled dilated bronchi. With varicose bronchiectasis, the bronchial lumen assumes a beaded configuration. Cystic bronchiectasis appears as a string of cysts caused by sectioning irregular dilated bronchi along their lengths, or a cluster of cysts, caused by multiple dilated bronchi lying adjacent to each other. Cluster of cysts are most commonly associated with an atelectatic lobe. Fluid levels, caused by retained secretions, may be present in the dependent portion of the dilated bronchi. Accumulation of secretions within bronchiectatic airways is typically recognizable as lobulated $\mathrm{V}$ - or Y-shaped structures (finger in glove sign). CT may show a completely collapsed lobe containing bronchiectatic airways. Subtle degrees of volume loss may present in lobes in relatively early disease.
Table 6.6 Specific causes of disseminated bronchiectasis

- Acute, chronic, or recurrent infections

- Genetic abnormalities

- Cystic fibrosis

- Dyskinetic (immotile) cilia syndrome

- Young syndrome

- Williams-Campbell syndrome

- Mounier-Kuhn syndrome (tracheobronchomegaly)

- Immunodeficiency syndromes

- Yellow nail syndrome

- Alpha-1-antitrypsin deficiency

- Noninfectious inflammatory diseases

- Allergic bronchopulmonary aspergillosis

- Asthma

- Systemic diseases (rheumatoid arthritis, Sjögren syndrome, systemic lupus erythematosus, inflammatory bowel disease)

- Post-transplantation bronchiolitis obliterans syndrome

Associated CT findings of bronchiolitis are present in about $70 \%$ of patients with bronchiectasis. These abnormalities are very common in patients with severe bronchiectasis and can even precede the development of bronchiectasis. The obstructive defect on pulmonary function testing in patients with bronchiectasis is the consequence of an obstructive involvement of the small airways (constrictive). The extent and severity of bronchiectasis and bronchial wall thickening correlate with airflow obstruction [14]. In patients with bronchiectasis, bronchial wall thickening and the extent of decreased lung attenuation are the strongest determinants of airflow obstruction. In addition, bronchial wall thickening on baseline CT correlates with functional deterioration overtime [15].

MDCT with thin collimation is the optimal imaging test to assess the presence and extent of bronchiectasis. Several studies have shown that multiplanar reformations increase the detection rate of bronchiectasis, readers' confidence as to the distribution of bronchiectasis, and agreement among observers as to the diagnosis of bronchiectasis.

The reliability of CT for distinguishing among the causes of bronchiectasis is somewhat controversial. An underlying cause for bronchiectasis is found in fewer than half of patients, and CT features alone do not usually allow confident distinction between idiopathic bronchiectasis and known causes of bronchiectasis (Table 6.6).

\subsection{Small Airway Diseases $[2,16]$}

Although normal bronchioles are below the resolution of thin-section CT, they can become detectable when inflammation of the airway wall and accompanying exudate develop. Some bronchiolar changes are too small to be visible directly but result in indirect signs on $\mathrm{CT}$. 


\subsubsection{Small Centrilobular Nodular and Branching Linear Opacities (Tree-in-Bud)}

Tree-in-bud opacities are defined as small centrilobular nodular and branching linear opacities, usually V- or Y-shaped, reflecting abnormally dilated bronchioles with thickened walls and mucus or exudate filling lumens. Associated peribronchiolar inflammation is often present, contributing to the CT appearance. Tree-in-bud opacities are characteristic of acute or chronic infectious bronchiolitis. They also occur in diffuse panbronchiolitis and aspiration.

\subsubsection{Poorly Defined Centrilobular Nodules}

Poorly defined centrilobular nodules reflect the presence of peribronchiolar inflammation in the absence of airway filling. When the distribution of nodules is diffuse and homogeneous, the pattern is suggestive of bronchiolar or vascular diseases. Bronchiolar disease associated with poorly defined centrilobular nodules includes respiratory bronchiolitis, hypersensitivity pneumonitis, and follicular bronchiolitis.

\subsubsection{Decreased Lung Attenuation and Mosaic Perfusion}

Areas of decreased lung attenuation associated with decreased vessel calibre on $\mathrm{CT}$ reflect bronchiolar obstruction and associated reflex vasoconstriction. In acute bronchiolar obstruction, decreased perfusion represents a physiologic reflex of hypoxic vasoconstriction, whereas irreversible vascular remodelling occurs with chronic bronchiolar obstruction. The areas of decreased lung attenuation related to hypoperfusion can be patchy or widespread. They are poorly defined or sharply demarcated giving a geographical outline, representing a collection of affected pulmonary lobules. Redistribution of blood flow to the normally ventilated areas causes localized increased attenuation. The patchwork of abnormal areas of low attenuation and normal lung or less diseased areas results in mosaic attenuation on CT, also termed mosaic perfusion. Expiratory CT accentuates the pattern of mosaic attenuation where areas of air trapping remain low in attenuation, whereas normal lung increases in attenuation. Usually the regional heterogeneity of lung attenuation is apparent at full inspiration.

Key Point

- MiniP is helpful in detecting mosaic attenuation.
However, with more extensive air trapping, the lack of regional homogeneity of lung attenuation can be challenging to detect on inspiratory scans, and, as a result, mosaic attenuation becomes visible only on expiratory scans. In patients with particularly severe and widespread involvement of the small airways, the patchy distribution of hypoattenuation and mosaic pattern is lost. Inspiratory scans show an apparent uniformity of decreased attenuation in the lungs and scans obtained at end expiration may appear similar to inspiration scan. In these patients, the most striking features are paucity of pulmonary vessels and lack of change of the cross-sectional area of lung between inspiration and expiration. Mosaic attenuation may be seen in patients who have constrictive bronchiolitis, bronchiolitis associated with hypersensitivity pneumonitis, asthma, and COPD.

\subsubsection{Expiratory Air Trapping}

Lobular areas of air trapping may be readily apparent on expiratory CT but are occult on inspiratory CT. Foci of lobular air trapping are usually well-demarcated reflecting the geometry of individual or joined lobules. Lobular areas of air trapping may be present on expiratory CT scans of normal individuals, especially in the medial and posterior basal and apical portions of the superior segments of the lower lobes. However, when lobular air trapping is present in the nondependent portions of the lung or the overall extent is equal or greater than one segment, air trapping should be considered as abnormal. Expiratory air trapping occurs in smokers and in patients with asthma, constrictive bronchiolitis, and bronchiolitis associated with hypersensitivity pneumonitis and sarcoidosis.

\subsection{Constrictive (Obliterative) Bronchiolitis $[2,16]$}

Constrictive bronchiolitis, characterized by submucosal circumferential fibrosis along the central axis of terminal bronchioles, is the result of a variety of causes and, rarely, is idiopathic (Table 6.7).

The main thin-section CT findings usually consist of areas of decreased lung attenuation associated with vessels of decreased calibre on inspiratory scans and air trapping on expiratory scans. Bronchial wall thickening and bronchiectasis, both central and peripheral, are also commonly present.

The areas of decreased lung attenuation and perfusion may be confined to or predominant in one lung, particularly in Swyer-James or MacLeod syndrome, that is a variant form of postinfectious constrictive bronchiolitis in which the constrictive bronchiolar lesions affect predominantly one lung. 
Table 6.7 Causes of and association with obliterative (constrictive) bronchiolitis

- Postinfection

- Childhood viral infection (adenovirus, respiratory syncytial virus, influenza, parainfluenza)

- Adulthood and childhood (Mycoplasma pneumoniae, Pneumocystis jiroveci in AIDS patients, endobronchial spread of tuberculosis, bacterial bronchiolar infection)

- Post-inhalation (toxic fumes and gases)

- Diffuse aspiration bronchiolitis (chronic occult aspiration in the elderly, patients with dysphagia)

- Connective tissue disorders (rheumatoid arthritis, Sjögren syndrome)

- Allograft recipients (bone marrow transplant, heart-lung, or lung transplant)

- Drugs (penicillamine, lomustine)

- Ulcerative colitis

- Other conditions

- Bronchiectasis

- Cystic fibrosis

- Hypersensitivity pneumonitis

- Diffuse idiopathic pulmonary neuroendocrine cell hyperplasia (DIPNECH)

- Excessive Sauropus androgynus (katuk, sweet leaf, or star gooseberry) ingestion

- Idiopathic

\subsection{Asthma [17]}

The main clinical indication for imaging patients with asthma is to identify diseases that may mimic asthma clinically, particularly hypersensitivity pneumonitis, constrictive bronchiolitis, and tracheal or carinal obstruction by neoplastic or nonneoplastic tracheal disorders. CT of patients with asthma can be normal or shows bronchial abnormalities. Mucoid impaction and linear bands, reflecting subsegmental or segmental atelectasis, are reversible on follow-up. Bronchial wall thickening is commonly present and has been shown to correlate with clinical severity and duration of asthma and the degree of airflow obstruction. It also correlates with pathologic measures of remodelling from bronchial biopsies [18]. Bronchiectasis may also be present, and its extent is associated with an increased severity of asthma.

In patients with persistent moderate asthma, mosaic attenuation reflects remodelling in the small airways. The extent of expiratory air trapping does not change after inhalation of salbutamol. This allows to exclude the hypothesis of bronchoconstriction to explain gas trapping in these patients and to reinforce the role of airway remodelling. In contrast, in patients with mild or moderate uncontrolled asthma, therapy with inhaled corticosteroids reduces the degree of air trapping on CT, suggesting that CT can be used as a surrogate marker for assessing disease control.

\subsection{Airway Disease in COPD [19]}

Airway disease phenotypes of COPD include abnormalities of the small and large airways. Poorly defined centrilobular nodules primarily in the upper lobes reflect inflammatory changes in and around the bronchioles (respiratory bronchiolitis), which are reversible after smoking cessation and steroids. Mosaic attenuation and expiratory air trapping reflect remodelling of the small airways (obstructive bronchiolitis).

Bronchial wall thickening is frequently present. Bronchial wall thickness is one of the strongest determinants of FEV1 in patients with COPD. The measures of airway thickness are also associated with chronic bronchitis with bronchodilator responsiveness and with a paradoxical response to bronchodilators. In addition, CT studies have consistently showed reduced airway lumen dimensions and fewer peripheral airways in COPD.

Moderate tubular bronchiectasis can occur, particularly in the lower lobes, as a result of injury to cartilage. Bronchiectasis is often associated with more severe COPD exacerbations, lower airway bacterial colonization, and increased sputum inflammatory markers [20]. However, the presence of bilateral varicose and cystic bronchiectasis in patients with panlobular emphysema should raise the diagnosis of alpha-1-antitrypsin deficiency.

\subsection{Concluding Remarks}

Volumetric thin-collimation CT acquisition at full inspiration and expiration has tremendously improved the accuracy of imaging in the detection and characterization of airway disease. Both proximal and distal airway disease may be identified and correctly assessed in terms of extent and severity.

CT and endoscopy play complementary roles in the assessment of tracheobronchial lesions, including neoplasms, inflammation, and infection.

$\mathrm{CT}$ remains the imaging technique of reference in the diagnosis and extent assessment of bronchiectasis. It has gained much interest from the respiratory physicians in the assessment of obstructive lung disease, including COPD, chronic asthma, and constrictive bronchiolitis. 


\section{Take-Home Messages}

- Volumetric thin-collimation CT with multiplanar reformations along the long axis and strictly perpendicular to the central axis of the airways has improved detection, characterization, and extent assessment of any tracheal or bronchial lesion.

- MIP images are helpful to detect foci of inflammatory or infectious lesions in terminal bronchioles.

- Expiratory CT scan and minIP are helpful to detect gas trapping due to obstruction of the bronchiolar lumens.

- Dynamic expiratory CT images may offer feasible alternative to bronchoscopy in patients with suspected tracheobronchomalacia or expiratory dynamic airway collapse by showing complete relapse or collapse greater than $80 \%$ of airway lumen.
5. Kang EY. Large airway diseases. J Thorac Imaging. 2011;26:249-62.

6. Ngo AV, Walker CM, Chung JH, et al. Tumors and tumorlike conditions of the large airways. AJR Am J Roentgenol. 2013;201:301-13.

7. Grenier PA, Beigelman-Aubry C, Brillet PY. Nonneoplastic tracheal and bronchial stenoses. Radiol Clin N Am. 2009;47:243-60.

8. Murgu S, Colt H. Tracheobronchomalacia and excessive dynamic airway collapse. Clin Chest Med. 2013;34:527-55.

9. Lee EY, Litmanovich D, Boiselle PM. Multidetector CT evaluation of tracheobronchomalacia. Radiol Clin N Am. 2009;47:261-9.

10. Ridge CA, O'donnell CR, Lee EY, Majid A, et al. Tracheobronchomalacia: current concepts and controversies. J Thorac Imaging. 2011;26:278-89.

11. Sverzellati N, Ingegnoli A, Calabrò E, et al. Bronchial diverticula in smokers on thin-section CT. Eur Radiol. 2010;20:88-94.

12. Javidan-Nejad C, Bhalla S. Bronchiectasis. Radiol Clin N Am. 2009; 47:289-306.

13. Feldman C. Bronchiectasis: new approaches to diagnosis and management. Clin Chest Med. 2011;32:535-46.

14. Roberts HR, Wells AU, Milne DG, et al. Airflow obstruction in bronchiectasis: correlation between computed tomography features and pulmonary function tests. Thorax. 2000;55:198-204.

15. Sheehan RE, Wells AU, Copley SJ, et al. A comparison of serial computed tomography and functional change in bronchiectasis. Eur Respir J. 2002;20:581-7.

16. Pipavath SN, Stern EJ. Imaging of small airway disease (SAD). Radiol Clin N Am. 2009;47:307-16.

17. Kauczor HU, Wielpütz MO, Owsijewitsch M, et al. Computed tomographic imaging of the airways in COPD and asthma. J Thorac Imaging. 2011;26:290-300.

18. Aysola RS, Hoffman EA, Gierada D, et al. Airway remodeling measured by multidetector CT is increased in severe asthma and correlates with pathology. Chest. 2008;134:1183-91.

19. Ley-Zaporozhan J, Kauczor HU. Imaging of airways: chronic obstructive pulmonary disease. Radiol Clin N Am. 2009;47:331-42.

20. Martínez-García MÁ, Soler-Cataluña JJ, Donat Sanz Y, et al. Factors associated with bronchiectasis in patients with COPD. Chest. 2011;140:1130-7.
4. Chung JH, Kanne JP, Gilman MD. CT of diffuse tracheal diseases. AJR Am J Roentgenol. 2011;196:W240-8. technique and normal results. Radiol Clin N Am. 2009;47:185-201.

2. Grenier PA, Beigelman-Aubry C, Fetita C, et al. New frontiers in CT imaging of airway disease. Eur Radiol. 2002;12:1022-44.

3. Ferretti GR, Bithigoffer C, Righini CA, et al. Imaging of tumors of the trachea and central bronchi. Radiol Clin N Am. 2009;47:227-41.

Open Access This chapter is licensed under the terms of the Creative Commons Attribution 4.0 International License (http://creativecommons. org/licenses/by/4.0/), which permits use, sharing, adaptation, distribution and reproduction in any medium or format, as long as you give appropriate credit to the original author(s) and the source, provide a link to the Creative Commons license and indicate if changes were made.

The images or other third party material in this chapter are included in the chapter's Creative Commons license, unless indicated otherwise in a credit line to the material. If material is not included in the chapter's Creative Commons license and your intended use is not permitted by statutory regulation or exceeds the permitted use, you will need to obtain permission directly from the copyright holder. 